\title{
Pd-Catalyzed Microwave Irradiated Regioselective Aroylation Reaction of Crotyl- and Allyltrifluoroborates
}

\author{
Mohammad Al-Masum*, Sarah Legan, Kwei-Yu Liu \\ Department of Chemistry, Tennessee State University, Nashville, TN, USA \\ Email: *malmasum@tnstate.edu
}

How to cite this paper: Al-Masum, M., Legan, S. and Liu, K.-Y. (2016) Pd-Catalyzed Microwave Irradiated Regioselective Aroylation Reaction of Crotyl- and Allyltrifluoroborates. International Journal of Organic Chemistry, 6, 220-232.

http://dx.doi.org/10.4236/ijoc.2016.64022

Received: September 16, 2016

Accepted: December 17, 2016

Published: December 20, 2016

Copyright (c) 2016 by authors and Scientific Research Publishing Inc. This work is licensed under the Creative Commons Attribution International License (CC BY 4.0)

http://creativecommons.org/licenses/by/4.0/

\begin{abstract}
An interesting regioselectivity is observed when the mixture of potassium crotyltrifluoroborate (1a) and aroyl chlorides having electron-deficient and electron-rich groups is microwaved in the presence of palladium-catalyst. In the case of electron withdrawing group with phenyl ring of aroyl chlorides, isomerized $\alpha, \beta$-unsaturated compound 3 is the product whereas electron donating group with phenyl ring of aroyl chlorides furnishes $\alpha$-adduct 4 . Similar aroylation reaction is also established for potassium allyltrifluoroborate (1b). In this case, regioselectivity is unaffected with changing electron-rich or electron-deficient groups in phenyl ring of the aroyl chlorides. Reactions proceed with, essentially in same rate, affording the corresponding aryl propenyl ketones (crotonophenones) 5 in good to high yields.
\end{abstract}

\section{Keywords}

Direct Aroylation, Regioselectivity, Stereoselectivity, Microwave

\section{Introduction}

One of the major transformations in organic chemistry is carbon-carbon bond formation through allylation reaction. The most common source for this type of reaction is allyl-M species where $\mathrm{M}$ is transition metal, $\mathrm{Mg}, \mathrm{Sn}, \mathrm{Si}, \mathrm{B}$ etc. [1]-[9]. Transition metal catalyzed cross-coupling reaction of allyl metal with aroyl chloride is rarely deliberated [9]. Organoboron reagent is extensively studied as a cross-coupling partner in Suzuki crosscoupling reaction. Because of the sensitivity and high reactivity of the allylboronic acid and allylboronic ester, they are essentially not used in cross-coupling reaction. Crotyl boronic acid and ester also show high reactivity. On the other hand, potassium allyltrifluoroborate and crotyltrifluoroborate are stable enough to use in reaction. The allyltrifluo- 
roborate salt formed either from allylboronic acid or allylboronic ester is air- and water-stable effective organoboron reagent for cross-coupling chemistry. Similar process is used to synthesize crotyltrifluoroborate. The growing emphasis of their use in Michael reaction [10]-[14], cross-coupling reaction [15]-[30] has interested us to explore the direct aroylation chemistry of potassium crotyl- and allyltrifluoroborates.

\section{Results and Discussion}

We found a noticeable regioselectivity when the mixture of crotyltrifluoroborate and aroyl chlorides having electron-deficient and electron-rich groups was microwaved in the presence of palladium-catalyst (Scheme 1). $\mathrm{PdCl}_{2}$ (d'bpf) complex and its active use as a catalyst in various organic transformations involving potassium allyltrifluoroborate, potassium styryltrifluoroborates, and potassium aryltrifluoroborates in our laboratory inspire us to embrace cross-coupling chemistry in new reactions and methodology. It is well documented by X-ray structure that $\mathrm{PdCl}_{2}$ (dtbpf) has shown the largest P-Pd-P bite angle $\left(104.22^{\circ}\right)$ for a ferrocenyl biphosphine ligand [31]-[34] compared to the other ferrocenyl biphosphine ligands such as $\mathrm{PdCl}_{2}$ (dippf) $\left[\theta=103.59^{\circ}\right], \mathrm{PdCl}_{2}$ (dCypf) $\left[\theta=102.45^{\circ}\right], \operatorname{PdCl}_{2}(\mathrm{dmpf})\left[\theta=99.3^{\circ}\right], \operatorname{PdCl}_{2}$ (dppf) $\left[\theta=97.98^{\circ}\right]$. The high bite angle $\mathrm{PdCl}_{2}\left(\mathrm{~d}^{\mathrm{t}} \mathrm{bpf}\right)$ shows may be the reason of high catalyst effect in organic transformations by cross-coupling reaction.

The cross-coupling reaction between crotyltrifluoroborate 1 and aroyl chlorides 2 with electron-deficient group in the phenyl rings are summarized in Table 1 . When 4-trifluoromethoxy-benzoyl chloride (2a), 2-trifluoromethoxy-benzoyl chloride (2b), 2,4,5-trifluoro-benzoyl chloride (2c), cross-coupled with crotyltrifluoroborate 1a under microwave heating at $140^{\circ} \mathrm{C}$ for $20 \mathrm{~min}$ all gave the isolated corresponding $(E)$ 2-methyl-1-aryl-but-2-en-1-ones $\mathbf{3 a}, \mathbf{3 b}$, and $3 \mathbf{c}$ respectively in good yields (Table 1 ).<smiles>C/C=C/C[13C](C)C(C)(F)Br</smiles>

1a<smiles>C/C=C(\C)C(=O)c1ccc(OCC)cc1</smiles>

3

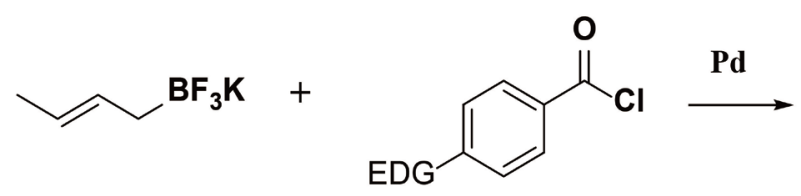

1a

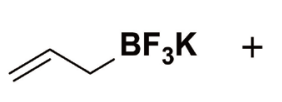

$1 b$<smiles>[Z]c1ccc(C(=O)Cl)cc1</smiles><smiles>C=CC(C)C(=O)c1ccc(OC(C)(C)C)cc1</smiles>

4<smiles>[Z]c1ccc(C(=O)/C=C/C)cc1</smiles>

5

Scheme 1. Regioselectivity. 
Table 1. Pd-catalyzed cross-coupling reaction of croty $\mathrm{IBF}_{3} \mathrm{~K} 1 \mathrm{a}$ and aroyl chlorides with electron deficient group $\mathbf{2}^{\mathrm{a}}$.

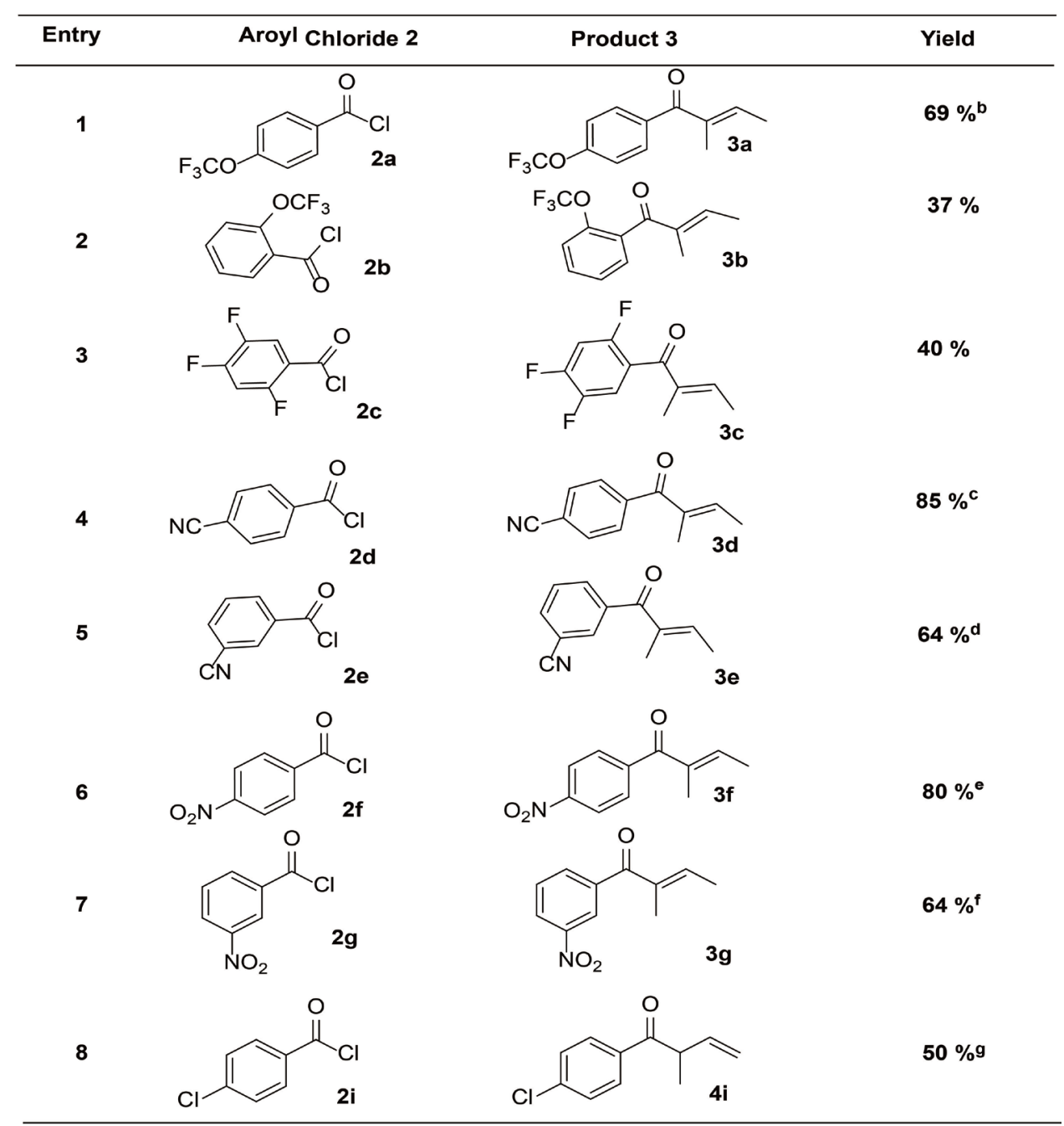

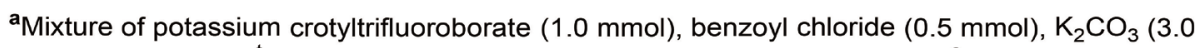
$\mathrm{mmol}$ ), and $\mathrm{PdCl}_{2}$ (d $\left.\mathrm{d}^{\mathrm{t}} \mathrm{bpf}\right)(0.015 \mathrm{mmol})$ in 1,4-dioxane was irradiated at $140{ }^{\circ} \mathrm{C}$ for $20 \mathrm{~min}$. All products are purified by Preparative TLC method. ${ }^{b}$ Trace amount of $\alpha$-selectivity product was obtained. "The ratio of $E$-product $3 \mathbf{d}: \alpha$-product is $2: 1$. ${ }^{\mathrm{d}}$ The ratio of $E$-product $3 \mathrm{e}: \alpha$-product is 10:1. 'Major product is $Z$-product $\mathbf{3} \mathbf{f}^{\prime}$. The ratio of $E: Z$ is $1: 4$. 'The ratio $E$-product $\mathbf{3 g}: Z$-product is 3:1. gNo isomerization except $\alpha$-product $4 \mathbf{i}$.

Entries 1 - 3). In case of 4-cyano-benzoyl chloride (2d) the corresponding crosscoupling product $\mathbf{3 d}$ along with $\alpha$-adduct $\mathbf{4 d}$ attained in 2:1 ratio. Interestingly, 3-cyano-benzoyl chloride (2e) gave mostly isomerized $(E)$-product 3e. Both cyano-aroyl chlorides formed products in decent yields (Entries 4,5 ). The regioselectivity and stereoselectivity of these reaction products are remarkable. When we run reaction with 4-nitrobenzoyl chloride (2f), major product was $(Z)$-isomer along with minor $E$-isomer with ratio $(\mathrm{E}: Z=1: 4)$. Again, when we run reaction with 3-nitrobenzoyl chloride $(2 \mathrm{~g})$, major product was $(E)$-isomer along with minor $Z$-isomer with ratio (E:Z = 3:1). All electron withdrawing moiety with phenyl ring enhanced isomerization to form predominantly $(E)$-1-aryl-2-methylbut-2-en-1-one 3 but 4-chloro-benzoyl chloride (2i) 
formed $\alpha$-product $4 \mathrm{i}$ only.

The reaction was performed on a $0.5 \mathrm{mmol}$ scale. After purging with argon a microwave reaction tube with stirrer bar was loaded with $83.0 \mathrm{mg}$ ( $0.5 \mathrm{mmol}, 98 \%$ pure) of potassium crotyltrifluoroborate $(1 \mathrm{a}), 0.157 \mathrm{~mL}(1.0 \mathrm{mmol})$ of aroyl chloride $2 \mathrm{a}, 414.0$ $\mathrm{mg}(3.0 \mathrm{mmol})$ of potassium carbonate, and $10.0 \mathrm{mg}(0.015 \mathrm{mmol})$ of $\mathrm{PdCl}_{2}\left(\mathrm{~d}^{\mathrm{t} b p f}\right)$. The reaction tube was then capped and flushed with argon following which $2.0 \mathrm{~mL}$ of 1,4-dioxane was added. The resulting reaction mixture was then microwaved at $140^{\circ} \mathrm{C}$ for $20 \mathrm{~min}$ in a microwave $(300 \mathrm{~W})$. Crude reaction product was extracted from inorganic material using dichloromethane. For purification, the crude product was subjected to preparative TLC using hexane/ethyl acetate (25/1) as eluent and collected the pure product.

In Table 2, results of the palladium-catalyzed cross-coupling reaction of crotyltrifluoroborate (1a) and aroyl chlorides having electron donating groups in phenyl rings are shown. In all cases, $\alpha$-regioselectivity was observed exclusively. It is clear that electron deficient phenyl moiety of the aroyl chlorides favor isomerization except in the case of 4-chlorobenzoyl chloride (Entry 8, Table 1) and electron donating phenyl moiety of the aroyl chloride obtained $\alpha$-selectivity without isomerization. Electron withdrawing group of the phenyl moiety enhance the isomerization because carbonyl carbon becomes more electrophilic and favors isomerization toward more stable $\alpha, \beta$-unsaturated carbonyl structure. The new result of microwave irradiated aroylation of crotyltrifluoroborate is an expansion of our earlier development in aroylation of allyltrifluoroborate.

Table 2. Pd-catalyzed cross-coupling reaction of crotyltrifluoroborate 1a and aroyl chlorides having electron donating group $2^{\mathrm{a}}$.

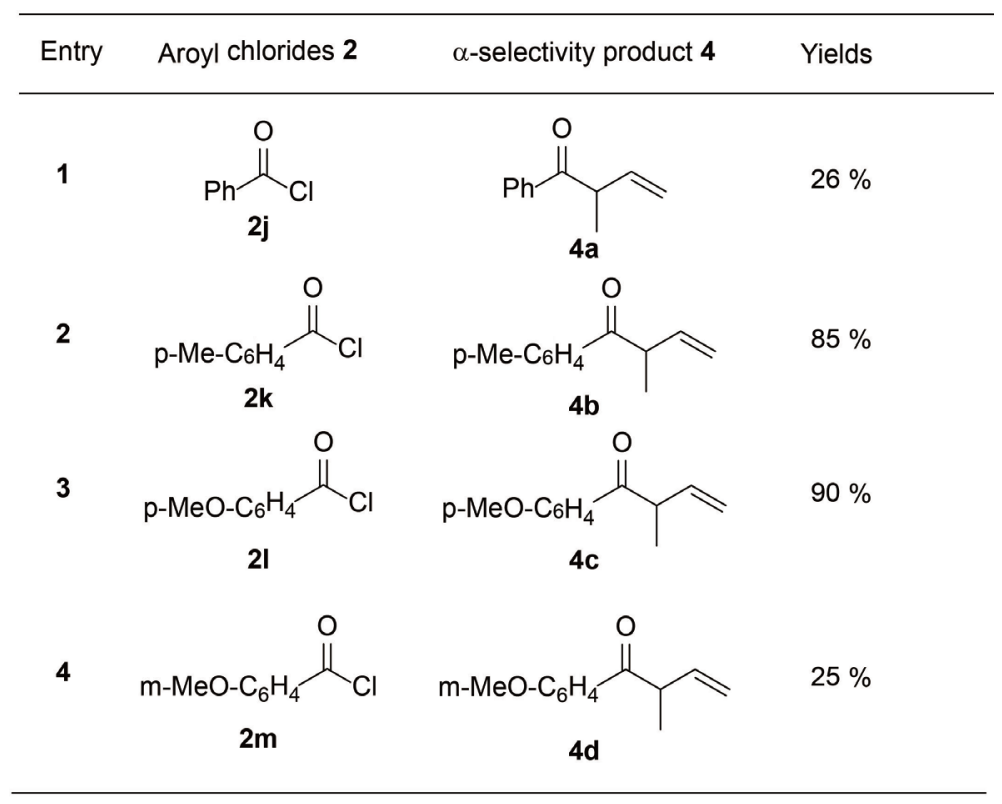

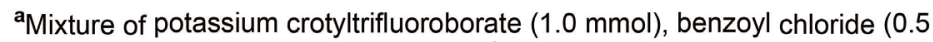
$\mathrm{mmol}), \mathrm{K}_{2} \mathrm{CO}_{3}(3.0 \mathrm{mmol})$, and $\mathrm{PdCl}_{2}$ ( $\left.\mathrm{d}^{\mathrm{t}} \mathrm{bpf}\right)(0.015 \mathrm{mmol})$ in 1,4-dioxane was irradiated at $1400^{\circ} \mathrm{C}$ for $20 \mathrm{~min}$. Yields are isolated pure products by column chromatography. 
[2] On changing electron-rich or electron-deficient groups in phenyl ring of the aroyl chlorides, the reaction between potassium allyltrifluoroborate (1b) and aroyl chlorides proceeded essentially in same rate affording the corresponding aryl propenyl ketones (crotonophenones) 5 in good to high yields (Table 3, entries 1-6, and entries 9, 10). In case of para-bromo-aroyl chloride (2o) and allyltrifluoroborate (1b), the cross-coupling product $\mathbf{5 g}$ formed as a minor product (Table 3 , entry 7 ) and homo-coupling product 4,4'-dibromobenzil (7) was the major one. Probably, possible palladium insertion to

Table 3. Palladuim-catalyzed cross-coupling reaction of allyltrifluoroborate $\mathbf{1 b}$ and aroyl chlorides $\mathbf{2}^{\mathrm{a}}$.

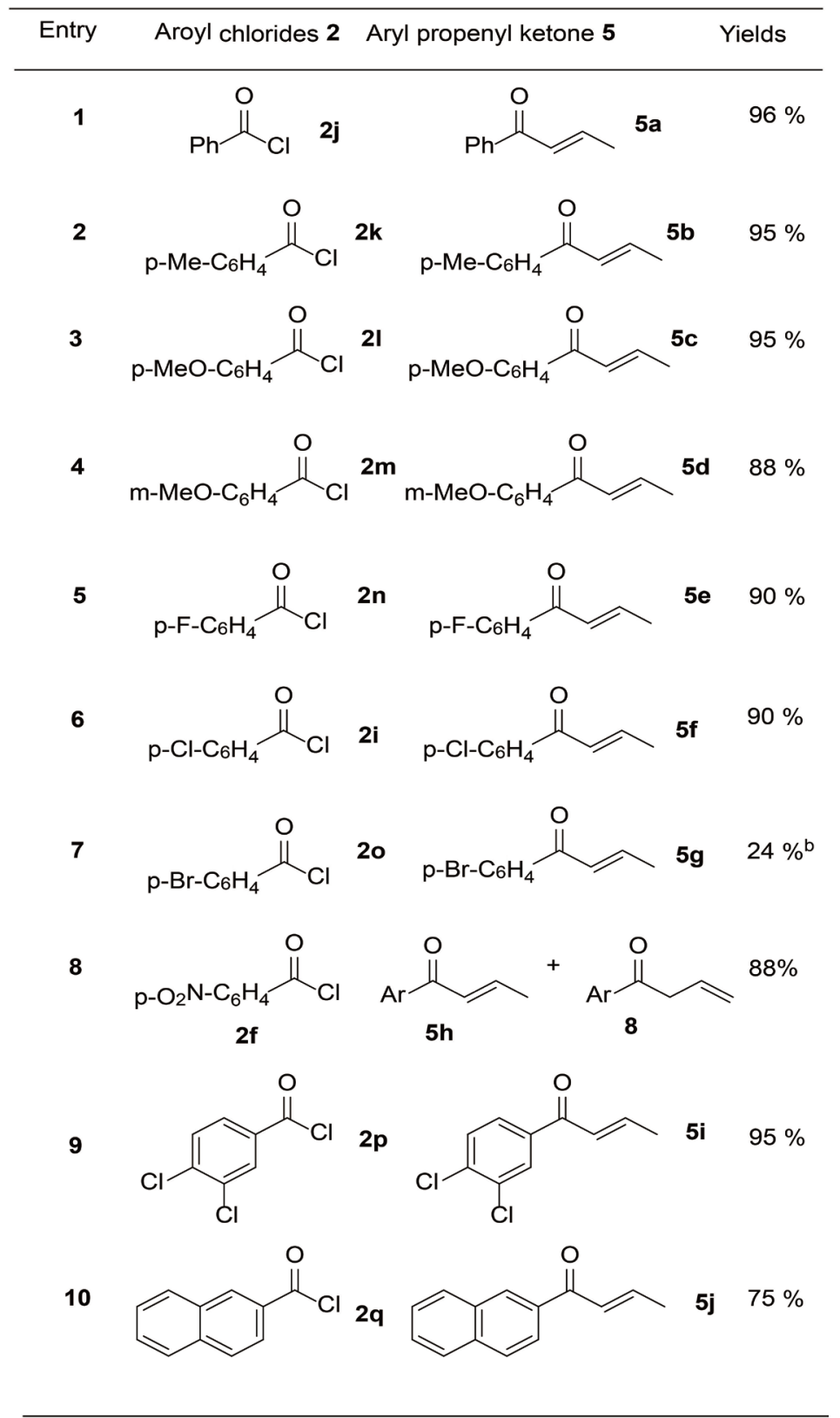

aYields are isolated pure products by column chromatography.

${ }^{\mathrm{b}} 4,4$ '-dibromobenzil (7) was the major product. 
bromide inhibits the transmetallation process and led to the homo-coupling product. Interestingly, when 4-nitro-aroyl chloride (2f) reacted with potassium allyltrifluoroborate (1b) under same reaction conditions, 50:50 mixture of $\beta$-adduct and $\gamma$-adduct were obtained (Table 3, entry 8 ) in good yields. Compare to other bases, potassium carbonate was most effective for this transformation. This new cross-coupling reaction requires excess amount of potassium carbonate. Control experiments without potassium carbonate did not give any product. Also, by adding a stoichiometric amount of potassium carbonate had very little effect of forming the desired product.

In this article, direct aroylation reaction of potassium crotyltrifluoroborate with aroyl chlorides and their regioselectivity have been successfully established. The probable catalytic cycle proposed for this aroylation reaction (Scheme 2) is based on regioselective reaction products. The oxidative addition of aroyl chloride to a palladium-complex yielding ArCO-Pd-Cl is new entity for cross-coupling chemistry. Very few reports demonstrated the direct cross-coupling reaction of aryl metal with aroyl chloride in the presence of palladium-catalyst [35]-[41].

The palladium inserted aroyl chloride, ArCOPdCl displays an excellent chemoselectivity in cross-coupling reaction when treated with potassium crotyl- and allyltrifluoroborate. Initially, we speculated that central carbon selectivity appears to be accomplished through palladacycle [42]. But mixture of products observed in the case of 4-nitro-aroyl chloride and allyltrifluoroborate (Table 3, entry 8) convince us to propose that the nucleophile may attack the terminal carbon of the $\pi$-allyl intermediate and

In case of electron withdrawing group with phenyl ring of aroyl chlorides

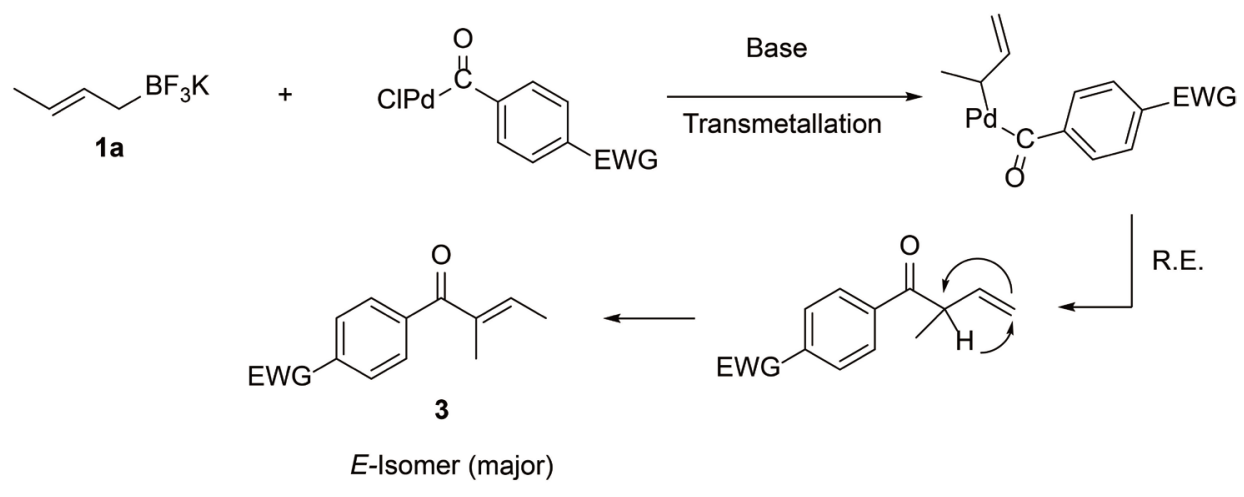

In case of electron donating group with phenyl ring of aroyl chlorides

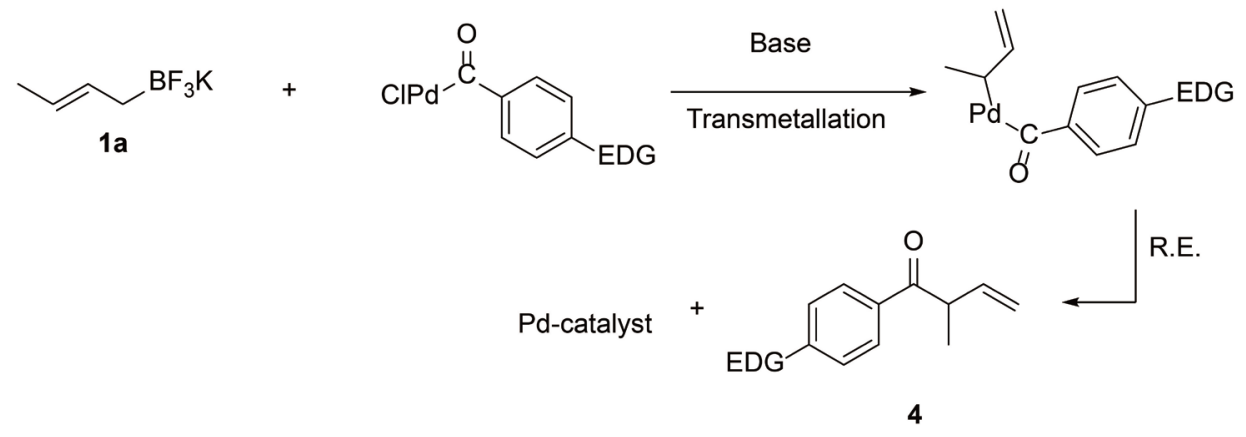

Scheme 2. Probable mechanism. 


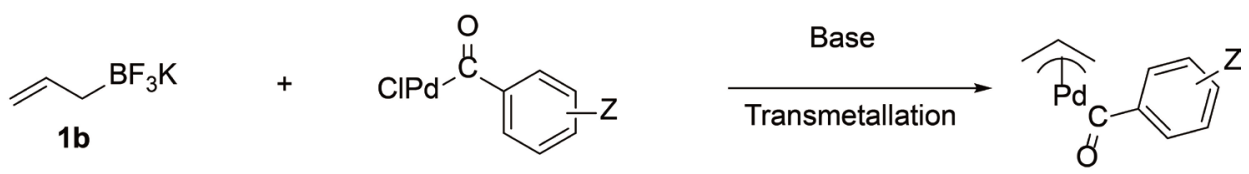<smiles>[Z]c1ccc(C(=O)/C=C/C)cc1</smiles>

OR<smiles>[Z]c1ccc(C(=O)OCC)cc1</smiles><smiles>[Z]c1ccc(C(=O)C2C[Te]CC2CCCO)cc1</smiles><smiles>[Z]c1ccc(C(=O)/C=C/C)cc1</smiles>

Scheme 3. In case of potassium allyltrifluoroborate.

subsequently isomerizes to the more stable $\alpha, \beta$-unsaturated ketone (Scheme 3 ). This new reaction methodology of cross-coupling reaction of potassium allyl-and crotyltrifluoroborates with aroyl chlorides is of great interest in organic synthesis and process chemistry. Further work is looked-for to establish the mechanism. Potassium allyl- and crotyltrifluoroborates are also applying to get allylamines and crotylamines in our laboratory.

\section{Procedure}

(E)-2-methyl-1-aryl-2-buten-1-ones (3a) from the cross-coupling of potassium crotyltrifluoroborate (1a) and 4-trifluoromethoxy-benzoyl chloride (2a) is shown as a representative procedure. The reaction was performed on a $0.5 \mathrm{mmol}$ scale. After purging with argon, a microwave reaction tube with stirrer bar was loaded with $83.0 \mathrm{mg}$ ( 0.5 $\mathrm{mmol}, 98 \%$ purity) of potassium crotyltrifluoroborate, $0.157 \mathrm{~mL}(1.0 \mathrm{mmol})$ of 4 -trifluoromethoxy-benzoyl chloride, $414.0 \mathrm{mg}(3.0 \mathrm{mmol})$ of potassium carbonate, and 10.0 $\mathrm{mg}(0.015 \mathrm{mmol})$ of $\mathrm{PdCl}_{2}\left(\mathrm{~d}^{\mathrm{t}} \mathrm{bpf}\right)$. The reaction tube was then capped and flushed with argon following which $2.0 \mathrm{~mL}$ of 1,4-dioxane was added. The resulting reaction mixture was then microwaved at $140^{\circ} \mathrm{C}$ for $20 \mathrm{~min}$ in a microwave $(300 \mathrm{~W})$. Crude reaction product was extracted from inorganic material using dichloromethane. For purification the crude product was subjected to preparative TLC using the volume ratio of hexane/ ethyl acetate (25/1) as eluents and collected the $84.0 \mathrm{mg}(68 \%)$ pure product, and characterized by GC/MS (Saturn 2200 Benchtop GC/MS) and NMR (Varian $300 \mathrm{MHz}$ ). 
Compound 3a: LRMS: Calculated for $\mathrm{C}_{12} \mathrm{H}_{11} \mathrm{O}_{2} \mathrm{~F}_{3} \mathrm{M}^{+}$244. Found: $244 .{ }^{1} \mathrm{H}$ NMR $\left(\mathrm{CDCl}_{3}, 300 \mathrm{MHz}\right) \delta 7.58(\mathrm{~m}, 2 \mathrm{H}$, Aromatic), $7.18(\mathrm{~m}, 2 \mathrm{H}$, aromatic), 6.31 (q, J = 6.9 $\mathrm{Hz}, 1 \mathrm{H}), 1.88\left(\mathrm{~s}, 3 \mathrm{H}, \mathrm{CH}_{3}\right), 1.81\left(\mathrm{~d}, \mathrm{~J}=6.6 \mathrm{~Hz}, 3 \mathrm{H}, \mathrm{CH}_{3}\right) ;{ }^{13} \mathrm{C} \mathrm{NMR}\left(\mathrm{CDCl}_{3}, 75.5 \mathrm{MHz}\right)$ $\delta 197.2,141.9,137.5,137.1,130.9,130.5,120.1,14.7,14.1 ;{ }^{19} \mathrm{~F}\left(\mathrm{CDCl}_{3}, 300 \mathrm{MHz}\right)$ $\delta$-57.7.

Compound 3b: LRMS: Calculated for $\mathrm{C}_{12} \mathrm{H}_{11} \mathrm{O}_{2} \mathrm{~F}_{3} \mathrm{M}^{+}$244. Found: 244. ${ }^{1} \mathrm{H}$ NMR $\left(\mathrm{CDCl}_{3}, 300 \mathrm{MHz}\right) \delta 7.35\left(\mathrm{~m}, 4 \mathrm{H}\right.$, Aromatic), $6.33(\mathrm{q}, \mathrm{J}=6.6 \mathrm{~Hz}, 1 \mathrm{H}), 1.93\left(\mathrm{~s}, 3 \mathrm{H}, \mathrm{CH}_{3}\right)$, $1.89\left(\mathrm{~d}, \mathrm{~J}=6.9 \mathrm{~Hz}, 3 \mathrm{H}, \mathrm{CH}_{3}\right) ;{ }^{13} \mathrm{C} \mathrm{NMR}\left(\mathrm{CDCl}_{3}, 75.5 \mathrm{MHz}\right) \delta 167.2,144.4,130.8,129.4$, 126.4, 120.6, 15.1, 10.7; ${ }^{19} \mathrm{~F}\left(\mathrm{CDCl}_{3}, 300 \mathrm{MHz}\right) \delta$-57.2.

Compound 3c: LRMS: Calculated for $\mathrm{C}_{11} \mathrm{H}_{9} \mathrm{OF}_{3} \mathrm{M}^{+}$214. Found: $214 .{ }^{1} \mathrm{H}$ NMR $\left(\mathrm{CDCl}_{3}, 300 \mathrm{MHz}\right) \delta 7.06\left(\mathrm{~m}, 2 \mathrm{H}\right.$, Aromatic), $6.45(\mathrm{q}, \mathrm{J}=6.9 \mathrm{~Hz}, 1 \mathrm{H}), 1.93\left(\mathrm{~s}, 3 \mathrm{H}, \mathrm{CH}_{3}\right)$, $1.91\left(\mathrm{~d}, \mathrm{~J}=6.9 \mathrm{~Hz}, 3 \mathrm{H}, \mathrm{CH}_{3}\right) ;{ }^{13} \mathrm{C} \mathrm{NMR}\left(\mathrm{CDCl}_{3}, 75.5 \mathrm{MHz}\right) \delta 192.7,144.4,138.5,118.1$, $106.4,106.1,15.1,11.0 ;{ }^{19} \mathrm{~F}\left(\mathrm{CDCl}_{3}, 300 \mathrm{MHz}\right) \delta$-114.3, -129.6, -141.9.

Compound 3d: LRMS: Calculated for $\mathrm{C}_{12} \mathrm{H}_{11} \mathrm{NO} \mathrm{M}^{+}$185. Found: $186 .{ }^{1} \mathrm{H}$ NMR $\left(\mathrm{CDCl}_{3}, 300 \mathrm{MHz}\right) \delta 7.55(\mathrm{~m}, 4 \mathrm{H}), 6.38(\mathrm{q}, \mathrm{J}=6.9 \mathrm{~Hz}, 1 \mathrm{H}), 1.95(\mathrm{~s}, 3 \mathrm{H}), 1.92(\mathrm{~d}, \mathrm{~J}=6.9$ $\mathrm{Hz}, 3 \mathrm{H}) ;{ }^{13} \mathrm{C} \mathrm{NMR}\left(\mathrm{CDCl}_{3}, 75.5 \mathrm{MHz}\right) \delta 199.7,143.7,139.2,137.5,132.3,131.8,129.3$, 14.9, 11.7.

Compound 3e: LRMS: Calculated for $\mathrm{C}_{12} \mathrm{H}_{11} \mathrm{NO} \mathrm{M}^{+}$185. Found: $186 .{ }^{1} \mathrm{H}$ NMR $\left(\mathrm{CDCl}_{3}, 300 \mathrm{MHz}\right) \delta 7.71(\mathrm{~m}, 4 \mathrm{H}), 6.38(\mathrm{q}, \mathrm{J}=6.9 \mathrm{~Hz}, 1 \mathrm{H}), 1.96(\mathrm{~s}, 3 \mathrm{H}), 1.92(\mathrm{~d}, \mathrm{~J}=6.9$ $\mathrm{Hz}, 3 \mathrm{H}) ;{ }^{13} \mathrm{C} \mathrm{NMR}\left(\mathrm{CDCl}_{3}, 75.5 \mathrm{MHz}\right) \delta 196.3,143.2,139.8,137.4,134.2,132.5,129.1$, $118.1,112.3,14.9,11.8$.

Compound 3f: LRMS: Calculated for $\mathrm{C}_{11} \mathrm{H}_{11} \mathrm{NO}_{3}(\mathrm{M}+2)^{+}$207. Found: 207. ${ }^{1} \mathrm{H}$ NMR (Acetone-d $\left.\mathrm{d}_{6}, 300 \mathrm{MHz}\right) \delta 8.24(\mathrm{~m}, 4 \mathrm{H}), 5.98(\mathrm{q}, \mathrm{J}=7.2 \mathrm{~Hz}, 1 \mathrm{H}), 1.96(\mathrm{~s}, 3 \mathrm{H}), 1.49(\mathrm{~d}, \mathrm{~J}=$ $7.2 \mathrm{~Hz}, 3 \mathrm{H}$ ); ${ }^{13} \mathrm{C}$ NMR (Acetone- $\left.\mathrm{d}_{6}, 75.5 \mathrm{MHz}\right) \delta 198.8,144.7,142.7,136.5,130.9,132.5$, $124.0,21.0,16.0,11.8$.

Compound 3g: LRMS: Calculated for $\mathrm{C}_{11} \mathrm{H}_{11} \mathrm{NO}_{3}(\mathrm{M}+2)^{+}$207. Found: 207. ${ }^{1} \mathrm{H} \mathrm{NMR}$ (Acetone-d $\left.\mathrm{d}_{6}, 300 \mathrm{MHz}\right) \delta 8.4-7.7(\mathrm{~m}, 4 \mathrm{H}), 6.47(\mathrm{q}, \mathrm{J}=5.4 \mathrm{~Hz}, 1 \mathrm{H}), 1.92(\mathrm{~s}, 3 \mathrm{H}), 1.90$ $(\mathrm{m}, 3 \mathrm{H}) ;{ }^{13} \mathrm{C}$ NMR (Acetone- $\left.\mathrm{d}_{6}, 75.5 \mathrm{MHz}\right) \delta 196.3,143.9,138.0,135.7,130.6,128.3$, $126.4,124.4,15.0,12.0$.

The reaction procedure used for compound 3 was also applied for the synthesis of $\alpha$-selectivity products 4 . Instead of preparative TLC, silica gel chromatography was applied for purification.

Spectral data: Compound 4a: LRMS: Calculated for $\mathrm{C}_{11} \mathrm{H}_{12} \mathrm{O} \mathrm{M}^{+}$160. Found: $160 .{ }^{1} \mathrm{H}$ NMR $\left(\mathrm{CDCl}_{3}, 300 \mathrm{MHz}\right) \delta 8.13-7.49(\mathrm{~m}, 5 \mathrm{H}$, Aromatic), $6.01(\mathrm{~m}, 1 \mathrm{H}), 5.20(\mathrm{~m}, 2 \mathrm{H}$, $\left.=\mathrm{CH}_{2}\right), 4.18(\mathrm{~m}, 1 \mathrm{H}) 1.34\left(\mathrm{~d}, \mathrm{~J}=6.6 \mathrm{~Hz}, 3 \mathrm{H}, \mathrm{CH}_{3}\right) ;{ }^{13} \mathrm{C} \mathrm{NMR}\left(\mathrm{CDCl}_{3}, 75.5 \mathrm{MHz}\right) \delta$ 210.0, 133.6, 132.9, 132.3, 130.1, 129.3, 129.1, 128.6, 116.5, 45.4, 17.0.

Compound 4b: LRMS: Calculated for $\mathrm{C}_{12} \mathrm{H}_{14} \mathrm{O} \mathrm{M}^{+}$174. Found: $174 .{ }^{1} \mathrm{H} \mathrm{NMR}\left(\mathrm{CDCl}_{3}\right.$, $300 \mathrm{MHz}) \delta 7.87-7.12(\mathrm{~m}, 4 \mathrm{H}$, Aromatic), $5.86(\mathrm{~m}, 1 \mathrm{H}), 5.18(\mathrm{~m}, 2 \mathrm{H}), 4.18(\mathrm{~m}, 1 \mathrm{H})$, $3.5(\mathrm{~s}, 3 \mathrm{H}), 1.3\left(\mathrm{~d}, \mathrm{~J}=7.2 \mathrm{~Hz}, 3 \mathrm{H}, \mathrm{CH}_{3}\right) ;{ }^{13} \mathrm{C} \mathrm{NMR}\left(\mathrm{CDCl}_{3}, 75.5 \mathrm{MHz}\right) \delta 201.1,143.7$, 143.7, 138.1, 133.5, 129.1, 129.8, 116.2, 58.0, 45.2, 21.4, 15.0.

Compound 4c: LRMS: Calculated for $\mathrm{C}_{12} \mathrm{H}_{14} \mathrm{O}_{2} \mathrm{M}^{+}$190. Found: $190 .{ }^{1} \mathrm{H}$ NMR $\left(\mathrm{CDCl}_{3}, 300 \mathrm{MHz}\right) \delta 8.6-6.9(\mathrm{~m}, 4 \mathrm{H}$, Aromatic), $5.99(\mathrm{~m}, 1 \mathrm{H}), 5.14(\mathrm{~m}, 2 \mathrm{H}), 4.18(\mathrm{~m}$, 
$1 \mathrm{H}), 3.87\left(\mathrm{~s}, \mathrm{H}_{3} \mathrm{CO}\right) ;{ }^{13} \mathrm{C} \mathrm{NMR}\left(\mathrm{CDCl}_{3}, 75.5 \mathrm{MHz}\right) \delta 199.7,162.5,139.1,131.8,130.8$, 128.8, 113.7, 113.0, 55.5, 31.5, 14.1 .

Compound 4d: LRMS: Calculated for $\mathrm{C}_{12} \mathrm{H}_{14} \mathrm{O}_{2} \mathrm{M}^{+}$190. Found: $190 .{ }^{1} \mathrm{H}$ NMR $\left(\mathrm{CDCl}_{3}, 300 \mathrm{MHz}\right) \delta 7.37(\mathrm{~m}, 4 \mathrm{H}), 6.0(\mathrm{~m}, 1 \mathrm{H}), 5.18(\mathrm{~m}, 2 \mathrm{H}), 4.18(\mathrm{~m}, 1 \mathrm{H}), 3.87(\mathrm{~s}, 3 \mathrm{H}$, $\left.\mathrm{OCH}_{3}\right), 1.33\left(\mathrm{~d}, \mathrm{~J}=6.9 \mathrm{~Hz}, 3 \mathrm{H}, \mathrm{CH}_{3}\right) ;{ }^{13} \mathrm{C} \mathrm{NMR}\left(\mathrm{CDCl}_{3}, 75.5 \mathrm{MHz}\right) \delta 201.3,160.2$, 138.3, 129.7, 122.8, 121.3, 120.5, 119.6, 116.7, 55.6, 45.6, 17.3.

Compound 4i: ${ }^{1} \mathrm{H}$ NMR $\left(\mathrm{CDCl}_{3}, 300 \mathrm{MHz}\right) \delta 7.95-7.41(\mathrm{~m}, 4 \mathrm{H}$, Aromatic), $5.97(\mathrm{~m}$, $1 \mathrm{H}), 5.17(\mathrm{~m}, 2 \mathrm{H}), 4.12(\mathrm{q}, \mathrm{J}=6.6 \mathrm{~Hz}, 1 \mathrm{H}), 1.33(\mathrm{t}, \mathrm{J}=5.1 \mathrm{~Hz}, 3 \mathrm{H}) ;{ }^{13} \mathrm{C} \mathrm{NMR}\left(\mathrm{CDCl}_{3}\right.$, $75.5 \mathrm{MHz}) \delta 199.9,139.3,137.7,134.4,129.9,128.8,116.8,113.0,45.5,16.9$.

Crotonophenone ( $5 \mathrm{a}$ ) from the cross-coupling of potassium allyltrifluoroborate (1b) and benzoyl chloride (2j) is shown as a representative procedure. A dry microwave pyrex tube was purged with argon. Potassium allyltrifluoroborate, $73.0 \mathrm{mg}(0.5 \mathrm{mmol})$, anhydrous potassium carbonate, $414 \mathrm{mg}(3.0 \mathrm{mmol}), \mathrm{PdCl}_{2}$ (dtbpf) catalyst, $9.9 \mathrm{mg}$ $(0.015 \mathrm{mmol})$, benzoyl chloride, $114 \mu \mathrm{L}(1.0 \mathrm{mmol})$ and a magnetic bar were packed in a microwave tube. The reactants were then flushed with argon for $1-2$ minutes to prevent the presence of air from causing the decomposition of the Pd catalyst followed by the addition of $2.0 \mathrm{~mL}$ of dry 1,4-dioxane from a sure-seal bottle. The resulting mixture in the microwave tube was subsequently inserted into the microwave (CEM, Discover) and heated at $140^{\circ} \mathrm{C}$ for $20 \mathrm{~min}$ at $300 \mathrm{~W}$. After removal from the microwave, the product mixture was extracted with diethyl ether and separated from the inorganic byproducts through filtration. The crude product was subjected to silica gel chromatography or preparative thin layer chromatography with hexane/ethyl acetate (25/1) as the eluent and the pure product was isolated, dried, and characterized by GC/MS (Saturn 2200 Benchtop GC/MS) and NMR (Varian $300 \mathrm{MHz}$ ).

Compound 5a: LRMS: Calculated for $\mathrm{C}_{10} \mathrm{H}_{10} \mathrm{O} \mathrm{M}^{+}$146. Found: $146 .{ }^{1} \mathrm{H} \mathrm{NMR}\left(\mathrm{CDCl}_{3}\right.$, $300 \mathrm{MHz}) \delta 7.96-7.45(\mathrm{~m}, 5 \mathrm{H}$, Aromatic), $7.09(\mathrm{~m}, 1 \mathrm{H}), 6.92(\mathrm{~m}, \mathrm{~J}=15.3 \mathrm{~Hz}, 1 \mathrm{H}), 2.01$ $\left(\mathrm{d}, \mathrm{J}=6.6 \mathrm{~Hz}, 3 \mathrm{H}, \mathrm{CH}_{3}\right) ;{ }^{13} \mathrm{C} \mathrm{NMR}\left(\mathrm{CDCl}_{3}, 75.5 \mathrm{MHz}\right) \delta 190.8,145.1,137.8,132.5$, 128.4, 127.4, 65.8, 30.2, 18.6.

Compound 5b: LRMS: Calculated for $\mathrm{C}_{11} \mathrm{H}_{12} \mathrm{O} \mathrm{M}{ }^{+}$160. Found: $160 .{ }^{1} \mathrm{H}$ NMR $\left(\mathrm{CDCl}_{3}\right.$, $300 \mathrm{MHz}) \delta 7.86-7.26(\mathrm{~m}, 4 \mathrm{H}$, Aromatic), $7.06(\mathrm{~m}, 1 \mathrm{H}), 6.92(\mathrm{~m}, \mathrm{~J}=15.3 \mathrm{~Hz}, 1 \mathrm{H}), 2.43$ $\left(\mathrm{s}, 3 \mathrm{H}, \mathrm{CH}_{3}\right), 2.00\left(\mathrm{~d}, \mathrm{~J}=5.4 \mathrm{~Hz}, 3 \mathrm{H}, \mathrm{CH}_{3}\right) ;{ }^{13} \mathrm{C} \mathrm{NMR}\left(\mathrm{CDCl}_{3}, 75.5 \mathrm{MHz}\right) \delta 190.2,144.5$, 130.1, 129.1, 128.6, 127.4, 30.2, 21.6.

Compound 5c: LRMS: Calculated for $\mathrm{C}_{11} \mathrm{H}_{12} \mathrm{O}_{2} \mathrm{M}^{+}$176. Found: $176 .{ }^{1} \mathrm{H}$ NMR $\left(\mathrm{CDCl}_{3}, 300 \mathrm{MHz}\right) \delta 7.97-6.94(\mathrm{~m}, 4 \mathrm{H}$, Aromatic), $7.06(\mathrm{~m}, 1 \mathrm{H}), 6.90(\mathrm{~m}, 1 \mathrm{H}), 3.88(\mathrm{~s}$, $\left.3 \mathrm{H}, \mathrm{OCH}_{3}\right), 2.00\left(\mathrm{~d}, \mathrm{~J}=6.6 \mathrm{~Hz}, 3 \mathrm{H}, \mathrm{CH}_{3}\right) ;{ }^{13} \mathrm{C} \mathrm{NMR}\left(\mathrm{CDCl}_{3}, 75.5 \mathrm{MHz}\right) \delta 189.2,163.4$, 144.2, 131.0, 130.9, 127.3, 55.7, 18.8 .

Compound 5d: LRMS: Calculated for $\mathrm{C}_{11} \mathrm{H}_{12} \mathrm{O}_{2} \mathrm{M}^{+}$176. Found: 176. ${ }^{1} \mathrm{H}$ NMR $\left(\mathrm{CDCl}_{3}, 300 \mathrm{MHz}\right) \delta 7.44-7.29(\mathrm{~m}, 4 \mathrm{H}$, Aromatic), 7.00(m, $1 \mathrm{H}), 6.81(\mathrm{~m}, \mathrm{~J}=15.3 \mathrm{~Hz}$. $1 \mathrm{H}), 3.79\left(\mathrm{~s}, 3 \mathrm{H}, \mathrm{OCH}_{3}\right), 1.93\left(\mathrm{~d}, \mathrm{~J}=5.7 \mathrm{~Hz}, 3 \mathrm{H}, \mathrm{CH}_{3}\right) ;{ }^{13} \mathrm{C} \mathrm{NMR}\left(\mathrm{CDCl}_{3}, 75.5 \mathrm{MHz}\right) \delta$ 190.4, 159.7, 145.1, 139.2, 129.4, 127.4, 121.0, 119.1, 112.7, 65.8, 55.4.

Compound 5e: LRMS: Calculated for $\mathrm{C}_{10} \mathrm{H}_{9} \mathrm{FO} \mathrm{M}^{+}$164. Found: $164 .{ }^{1} \mathrm{H} \mathrm{NMR}\left(\mathrm{CDCl}_{3}\right.$, $300 \mathrm{MHz}) \delta 8.12-7.87\left(\mathrm{~m}, 4 \mathrm{H}\right.$, Aromatic), $7.12(\mathrm{~m}, 1 \mathrm{H}), 1.94\left(\mathrm{~d}, \mathrm{~J}=6.6 \mathrm{~Hz}, 3 \mathrm{H}, \mathrm{CH}_{3}\right)$; 
${ }^{13} \mathrm{C} \mathrm{NMR}\left(\mathrm{CDCl}_{3}, 75.5 \mathrm{MHz}\right) \delta 189.2,168.6,165.2,164,145.5,133.6,127.2,125.2,66.1$, 41.5.

Compound 5f: LRMS: Calculated for $\mathrm{C}_{10} \mathrm{H}_{9} \mathrm{ClO} \mathrm{M}^{+}$180. Found: $182 .{ }^{1} \mathrm{H} \mathrm{NMR}$ $\left(\mathrm{CDCl}_{3}, 300 \mathrm{MHz}\right) \delta 7.89-7.43(\mathrm{~m}, 4 \mathrm{H}$, Aromatic), $7.10(\mathrm{~m}, 1 \mathrm{H}), 6.88(\mathrm{~m}, \mathrm{~J}=15.3 \mathrm{~Hz}$, $1 \mathrm{H}), 2.03\left(\mathrm{~d}, \mathrm{~J}=6.6 \mathrm{~Hz}, 3 \mathrm{H}, \mathrm{CH}_{3}\right) ;{ }^{13} \mathrm{C} \mathrm{NMR}\left(\mathrm{CDCl}_{3}, 75.5 \mathrm{MHz}\right) \delta 189.3,145.7,139.0$, 136.1, 129.9, 128.8, 127.0, 36.0, 34.6, 31.5.

Compound 5g: LRMS: Calculated for $\mathrm{C}_{10} \mathrm{H}_{9} \mathrm{ClO} \mathrm{M}^{+}$224. Found: $226 .{ }^{1} \mathrm{H} \mathrm{NMR}$ $\left(\mathrm{CDCl}_{3}, 300 \mathrm{MHz}\right) \delta 7.91-7.52(\mathrm{~m}, 4 \mathrm{H}$, Aromatic), $7.01(\mathrm{~m}, 1 \mathrm{H}), 6.79(\mathrm{~m}, \mathrm{~J}=15 \mathrm{Mz}$, $1 \mathrm{H}), 1.92\left(\mathrm{~d}, \mathrm{~J}=6.4 \mathrm{~Hz}, 3 \mathrm{H}, \mathrm{CH}_{3}\right) ;{ }^{13} \mathrm{C} \mathrm{NMR}\left(\mathrm{CDCl}_{3}, 75.5 \mathrm{MHz}\right) \delta 198.3,146.0,144.8$, $136.8,132.0,130.2,129.2,127.2,126.0,18.9$.

Compound 5h: LRMS: Calculated for $\mathrm{C}_{10} \mathrm{H}_{9} \mathrm{NO}_{3} \mathrm{M}^{+}$191. Found: $192 .{ }^{1} \mathrm{H} \mathrm{NMR}$ $\left(\mathrm{CDCl}_{3}, 300 \mathrm{MHz}\right) \delta 8.35-8.04(\mathrm{~m}, 4 \mathrm{H}$, Aromatic), $7.15(\mathrm{~m}, 1 \mathrm{H}), 6.89(\mathrm{~m}, 1 \mathrm{H}), 2.05(\mathrm{~d}$, $\left.\mathrm{J}=6.6 \mathrm{~Hz}, 3 \mathrm{H}, \mathrm{CH}_{3}\right) ;{ }^{13} \mathrm{C} \mathrm{NMR}\left(\mathrm{CDCl}_{3}, 75.5 \mathrm{MHz}\right) \delta 188.0,147.8,143.1,131.2,129.6$, $127.3,123.9,14.3$.

Compound 5i: LRMS: Calculated for $\mathrm{C}_{10} \mathrm{H}_{8} \mathrm{Cl}_{2} \mathrm{O} \mathrm{M}^{+}$214. Found: $216 .{ }^{1} \mathrm{H} \mathrm{NMR}$ $\left(\mathrm{CDCl}_{3}, 300 \mathrm{MHz}\right) \delta 8.01-7.55(\mathrm{~m}, 4 \mathrm{H}$, Aromatic), $7.13(\mathrm{~m}, 1 \mathrm{H}), 6.85(\mathrm{~m}, \mathrm{~J}=15.3 \mathrm{~Hz}$, $1 \mathrm{H}), 2.03\left(\mathrm{~d}, \mathrm{~J}=6.6 \mathrm{~Hz}, 3 \mathrm{H}, \mathrm{CH}_{3}\right) ;{ }^{13} \mathrm{C} \mathrm{NMR}\left(\mathrm{CDCl}_{3}, 75.5 \mathrm{MHz}\right) \delta 188.1,146.5,137.4$, $130.6,130.4,127.5,126.5,18.7$.

Compound 5j: LRMS: Calculated for $\mathrm{C}_{14} \mathrm{H}_{12} \mathrm{O} \mathrm{M}{ }^{+}$196. Found: $196 .{ }^{1} \mathrm{H} \mathrm{NMR}\left(\mathrm{CDCl}_{3}\right.$, $300 \mathrm{MHz}) \delta 7.94-7.03\left(\mathrm{~m}, 9 \mathrm{H}\right.$, Aromatic), $1.97\left(\mathrm{~d}, \mathrm{~J}=5.7 \mathrm{~Hz}, 3 \mathrm{H}, \mathrm{CH}_{3}\right) ;{ }^{13} \mathrm{C} \mathrm{NMR}$ $\left(\mathrm{CDCl}_{3}, 75.5 \mathrm{MHz}\right) \delta 190.7,14.2,135.6,135.4,132.7,130.2,129.7,128.7,128.5,128.4$, $128.0,127.6,126.9,18.9$.

\section{Acknowledgements}

Financial support from US department of education Title III grant, Tennessee State University is thankfully acknowledged.

\section{References}

[1] Marshall, J.A. (2000) Synthesis and Reactions of Allylic, Allenic, Vinylic, and Arylmetal Reagents from Halides and Esters via Transient Organopalladium Intermediates. Chemical Reviews, 100, 3163-3185. https://doi.org/10.1021/cr000003u

[2] Stille, J.K. (1985) In: Hartley, F.R. and Patai, S., Eds., The Chemistry of the Metal-Carbon, Vol. 2, John Wiley, New York, 625.

[3] Sakurai, H. and Hosomi, A. (1977) Chemistry of Organosilicon Compounds. 99. Conjugate Addition of Allylsilanes to .Alpha.,.Beta.-Enones. A New Method of Stereoselective Introduction of the Angular Allyl Group in Fused Cyclic .Alpha.,.Beta.-Enones. Journal of the American Chemical Society, 99, 1673-1675. https://doi.org/10.1021/ja00447a080

[4] Yanagisawa, A., Hibino, H., Nomura, N. and Yamamoto, H. (1993) Unprecedented Gamma-Selective Nucleophilic Substitution Reaction of Allylmetal Reagents: A New CrossCoupling of Diphenyl Phosphates with Allylic Grignard Reagents. Journal of the American Chemical Society, 115, 5879-5880. https://doi.org/10.1021/ja00066a088

[5] Corey, E.J., Semmelhack, M.F. and Hegedus, L.S. (1968) The Course of Allylic Coupling Reactions Involving Allylnickel Complexes. Journal of the American Chemical Society, 90, 2416-2417. https://doi.org/10.1021/ja01011a035 
[6] Hirai, A., Nakamura, M. and Nakamura, E. (2000) Mechanism of Addition of Allylmetal to Vinylmetal. Dichotomy between Metallo-Ene Reaction and Metalla-Claisen Rearrangement. Journal of the American Chemical Society, 122, 11791-11798.

https://doi.org/10.1021/ja002563g

[7] Trost, B.M. and Vranken, D.L.V. (1996) Asymmetric Transition Metal-Catalyzed Allylic Alkylations. Chemical Reviews, 96, 395.

[8] Yamamoto, Y. and Asao, N. (1993) Selective Reactions Using Allylic metals. Chemical Reviews, 93, 2207.

[9] Al-Masum, M. and Liu, K.-Y. (2011) A New Organic Transformation by Introducing Crotyl/Allyltrifluoroborates in Cross-Coupling Reaction with Aroyl Chlorides. Tetrahedron Letters, 52, 5090-5093.

[10] Lautens, M. and Maddess, M.L. (2004) Chemoselective Cross Metathesis of Bishomoallylic Alcohols: Rapid Access to Fragment A of the Cryptophycins. Organic Letters, 6, 1883-1886.

[11] Nowrouzi, F., Janetzko, J. and Batey, R.A. (2010) Indium-Promoted Chemo- and Diastereoselective Allylation of $\alpha, \beta$-Epoxy Ketones with Potassium Allyltrifluoroborate. Organic Letters, 12, 5490-5493. https://doi.org/10.1021/ol1023757

[12] Thadani, A.N. and Batey, R.A. (2002) A Mild Protocol for Allylation and Highly Diastereoselective Syn or Anti Crotylation of Aldehydes in Biphasic and Aqueous Media Utilizing Potassium Allyl- and Crotyltrifluoroborates. Organic Letters, 4, 3827-3830. https://doi.org/10.1021/ol026619i

[13] Solin, N., Wallner, O.A. and Szabo, K.J. (2005) Palladium Pincer-Complex Catalyzed Allylation of Tosylimines by Potassium Trifluoro(Allyl)Borates. Organic Letters, 7, 689-691. https://doi.org/10.1021/ol0475010

[14] Nakamura, H. and Shimizu, K. (2011) Catalytic Reactions of Bis- $\pi$-Allylpalladium Generated from Allyltrifluoroborate. Tetrahedron Letters, 52, 426-429. https://doi.org/10.1016/j.tetlet.2010.11.082

[15] Al-Masum, M. and Alam, S. (2009) Remarkable Regioselectivity in Microwave-Enhanced Palladium-Catalyzed Allylation Reaction Involving Allyltrifluoroborates and Aryl Halides. Tetrahedron Letters, 50, 5201-5204. https://doi.org/10.1016/j.tetlet.2009.06.134

[16] Yamamoto, Y., Takada, S. and Miyaura, N. (2006) $\Gamma$-Selective Cross-Coupling of Potassium Allyltrifluoroborates with Aryl and 1-Alkenyl Bromides Catalyzed by a $\mathrm{Pd}(\mathrm{OAc}) 2 / \mathrm{D}-t$-BPF Complex. Chemistry Letters, 35, 704-705. https://doi.org/10.1246/cl.2006.704

[17] Sebelius, S., Olsson, V.J., Wallner, O.A. and Szabo, K.J. (2006) Palladium-Catalyzed Coupling of Allylboronic Acids with Iodobenzenes. Selective Formation of the Branched Allylic Product in the Absence of Directing Groups. Journal of the American Chemical Society, 128, 8150-8151. https://doi.org/10.1021/ja062585o

[18] Molander, G.A. and Ham, J. (2006) Synthesis of Functionalized Organotrifluoroborates via Halomethyltrifluoroborates. Organic Letters, 8, 2031-2034. https://doi.org/10.1021/ol060375a

[19] Molander, G.A. and Figueroa, R. (2005) Organotrifluoroborates: Expanding Organoboron Chemistry. Aldrichchimica Acta, 38, 49-56.

[20] Molander, G.A. and Felix, L.A. (2005) Stereoselective Suzuki-Miyaura Cross-Coupling Reactions of Potassium Alkenyltrifluoroborates with Alkenyl Bromides. Journal of Organic Chemistry, 70, 3950-3956. https://doi.org/10.1021/jo050286w

[21] Tremblay-Morin, J-P., Raeppel, S. and Gaudette, F. (2004) Lewis Acid-Catalyzed Mannich type Reactions with Potassium Organotrifluoroborates. Tetrahedron Letters, 45, 3471-3474. https://doi.org/10.1016/j.tetlet.2004.03.014 
[22] Molander, G.A. and Ribagorda, M. (2003) Expanding Organoboron Chemistry: Epoxidation of Potassium Organotrifluoroborates. Journal of the American Chemical Society, 125, 11148-11149. https://doi.org/10.1021/ja0351140

[23] Quach, T.D. and Batey, R.A. (2003) Copper(II)-Catalyzed Ether Synthesis from Aliphatic Alcohols and Potassium Organotrifluoroborate Salts. Organic Letters, 5, 1381-1384. https://doi.org/10.1021/ol034454n

[24] Molander, G.A. and Bernardi, C.R. (2002) Suzuki-Miyaura Cross-Coupling Reactions of Potassium Alkenyltrifluoroborates. Journal of Organic Chemistry, 67, 8424-8429. https://doi.org/10.1021/jo026236y

[25] Molander, G.A., Katona, B.W. and Machrouhi, F. (2002) Development of the Suzuki-Miyaura Cross-Coupling Reaction: Use of Air-Stable Potassium Alkynyltrifluoroborates in Aryl Alkynylations. Journal of Organic Chemistry, 67, 8416-8423. https://doi.org/10.1021/jo0262356

[26] Pucheault, M., Darses, S. and Genet, J.-P. (2002) Potassium Organotrifluoroborates in Rhodium-Catalyzed Asymmetric 1,4-Additions to Enones. European Journal of Organic Chemistry, 2002, 3552-3557. https://doi.org/10.1002/1099-0690(200211)2002:21<3552::AID-EJOC3552>3.0.CO;2-4

[27] Molander, G.A. and Rivero, M.R. (2002) Suzuki Cross-Coupling Reactions of Potassium Alkenyltrifluoroborates. Organic Letters, 4, 107-109. https://doi.org/10.1021/ol0169729

[28] Batey, R.A., Thadani, A.N., Smil, D.V. and Lough, A. (2000) Diastereoselective Allylation and Crotylation Reactions of Aldehydes with Potassium Allyl- and Crotyltrifluoroborates under Lewis Acid Catalysis. Synthesis, 2000, 990-998. https://doi.org/10.1055/s-2000-6303

[29] Darses, S., Michauld, G. and Genet, J.-P. (1999) Potassium Organotrifluoroborates: New Partners in Palladium-Catalysed Cross-Coupling Reactions. European Journal of Organic Chemistry, 1999, 1875-1883. https://doi.org/10.1002/(SICI)1099-0690(199908)1999:8<1875::AID-EJOC1875>3.0.CO;2$\underline{W}$

[30] Darses, S. and Genet, J.-P. (2008) Potassium Organotrifluoroborates: New Perspectives in Organic Synthesis. Chemical Reviews, 108, 288-325. https://doi.org/10.1021/cr0509758

[31] Mann, G., Shelby, Q., Roy, A.H. and Hartwig, J. (2003) Electronic and Steric Effects on the Reductive Elimination of Diaryl Ethers from Palladium(II). Organometallics, 22, 27752789. https://doi.org/10.1021/om030230x

[32] Elsagir, A.R., Gassner, F., Gorls, H. and Dinjus, E. (2000) Bidentate Ferrocenylphosphines and Their Palladium(II)Dichloride Complexes-X-Ray Structural and NMR Spectroscopic Investigations and First Results of Their Characteristics in the Pd-Catalysed Cooligomerisation of 1,3-Butadiene with $\mathrm{CO}_{2}$. Journal of Organometallic Chemistry, 597, 139-145. https://doi.org/10.1016/S0022-328X(99)00670-1

[33] Bianchini, C., Meli, A., Overhauser, W., Parisel, S., Passaglia, E., Ciardelli, F., Gusev, O.V., Kal'sin, A.M. and Vologdin, N. (2005) Ethylene Carbonylation in Methanol and in Aqueous Media by Palladium(II) Catalysts Modified with 1,1'-Bis(Dialkylphosphino)Ferrocenes. Organometallics, 24, 1018-1030. https://doi.org/10.1021/om049109w

[34] Grasa, G.A. and Colacot, T.J. (2007) A-Arylation of Ketones Using Highly Active, Air-Stable (DtBPF) PdX $2(\mathrm{X}=\mathrm{Cl}, \mathrm{Br})$ Catalysts. Organic Letters, 9, 5489-5492. https://doi.org/10.1021/ol702430a

[35] Al-Masum, M., Ng, E. and Wai, M.C. (2011) Palladium-Catalyzed Direct Cross-Coupling of Potassium Styryltrifluoroborates and Benzoyl Chlorides-A One Step Method for Chalcone Synthesis. Tetrahedron Letters, 52, 1008-1010. https://doi.org/10.1016/j.tetlet.2010.12.085 
[36] Labadie, J.W. and Stille, J.K. (1983) Mechanisms of the Palladium-Catalyzed Couplings of Acid Chlorides with Organotin Reagents. Journal of the American Chemical Society, 105, 6129-6137. https://doi.org/10.1021/ja00357a026

[37] Milstein, D. and Stille, J.K. (1978) A General, Selective, and Facile Method for Ketone Synthesis from Acid Chlorides and Organotin Compounds Catalyzed by Palladium. Journal of the American Chemical Society, 100, 3636-3638. https://doi.org/10.1021/ja00479a077

[38] Urawa, Y. and Ogura, K. (2003) A Convenient Method for Preparing Aromatic Ketones from Acyl Chlorides and Arylboronic Acids via Suzuki-Miyaura Type Coupling Reaction. Tetrahedron Letters, 44, 271-273. https://doi.org/10.1016/S0040-4039(02)02501-7

[39] Haddach, M. and McCarthy, J.R. (1999) A New Method for the Synthesis of Ketones: The Palladium-Catalyzed Cross-Coupling of Acid Chlorides with Arylboronic Acid. Tetrahedron Letters, 40, 3109-3112. https://doi.org/10.1016/S0040-4039(99)00476-1

[40] Bumagin, N., Korolev, D.N., Bumagin, N. and Korolev, D.N. (1999) Synthesis of Unsymmetric Ketones via Ligandless Pd-Catalyzed Reaction of Acyl Chlorides with Organoboranes. Tetrahedron Letters, 40, 3057-3060. https://doi.org/10.1016/S0040-4039(99)00364-0

[41] Xin, B., Zhang, Y. and Cheng, K. (2007) The Surfactant-Promoted Cross-Coupling Reactions of Arylboronic Acids with Carboxylic Anhydrides or Acyl Chlorides in Water. Synthesis, 2007, 1970-1978. https://doi.org/10.1055/s-2007-983729

[42] Hegedus, L.S., Darlington, W.H. and Russell, C.E. (1980) Cyclopropanation of Ester Enolates by .Pi-Allylpalladium Chloride Complexes. Journal of Organic Chemistry, 45, 5193 5196. https://doi.org/10.1021/jo01313a034

Submit or recommend next manuscript to SCIRP and we will provide best service for you:

Accepting pre-submission inquiries through Email, Facebook, LinkedIn, Twitter, etc.

A wide selection of journals (inclusive of 9 subjects, more than 200 journals)

Providing 24-hour high-quality service

User-friendly online submission system

Fair and swift peer-review system

Efficient typesetting and proofreading procedure

Display of the result of downloads and visits, as well as the number of cited articles

Maximum dissemination of your research work

Submit your manuscript at: http://papersubmission.scirp.org/

Or contact ijoc@scirp.org 